\title{
OS NITRITOS SÃO IMPORTANTES NA GÊNESE DO ADENOCARCINOMA ASSOCIADO AO ESÔFAGO DE BARRETT?
}

\author{
Are the nitrites important in a genesis of the adenocarcinoma associated to the \\ Barrett esophagus?
}

\author{
Sergio Ferreira MÓDENA, Luciana Rodrigues MEIRELLES, Marina Rachel ARAÚJO
}

\begin{abstract}
ABCDDV/608
Modena SF, Meirelles LR, Araujo MR. Os nitritos são importantes na gênese do adenocarcinoma associado ao esôfago de Barrett ? ABCD Arq Brás Cir Dig 2008; 21(3):124-9

RESUMO - Racional - O esôfago de Barrett é complicação do refluxo gastroesofágico e predispõe ao surgimento do adenocarcinoma, sendo que vários fatores têm sido relacionados à sua etiopatogenia. Objetivo - Analisar o modelo experimental de refluxo duodenogastroesofágico e a ingestão de solução de nitrito de sódio na gênese do adenocarcinoma associado ao esôfago de Barrett. Métodos - Sessenta ratos machos Wistar foram divididos em quatro grupos, com 20 animais controles não operados (10 animais ingeriram somente água e 10 água mais solução de nitrito de sódio) e 40 animais submetidos à anastomose duodenogastroesofágica látero-lateral (20 animais ingeriram somente água e 20 animais ingeriram água mais solução de nitrito de sódio). A ocorrência de esofagite, metaplasia, displasia, esôfago de Barrett e adenocarcinoma associado foram analisados. A classificação de Viena para displasia e adenocarcinoma foi empregada na análise dos resultados. Resultados - Após 42 semanas de observação, nos animais operados sem ingestão de nitritos o esôfago de Barrett foi registrado em 26,3\% (5/19), enquanto no grupo operado associado à ingestão de nitritos, foi encontrado em $72,3 \%$ (13/18) dos animais, sendo que neste grupo também foi encontrado seis adenocarcinomas (33,3\%). Nenhum animal não operado apresentou o esôfago de Barrett. As categorias 2, 3 e 5 da classificação de Viena somente foram encontradas nos animais operados que ingeriram nitrito de sódio (66,7\%). Conclusão - A ingestão de nitrito de sódio associado ao refluxo duodenogastroesofágico têm importante participação na gênese do adenocarcinoma associado ao esôfago de Barrett.

DESCRITORES - Refluxo duodenogástrico. Nitritos. Esôfago de Barrett. Adenocarcinoma.
\end{abstract}

\section{INTRODUÇÃO}

O Esôfago de Barrett (EB) é complicação da doença do refluxo gastro-esofágico (DRGE) e consiste em processo de substituição do epitélio escamoso do esôfago por colunar intestinal contendo células caliciformes, conhecido por metaplasia intestinal. É condição pré-maligna, acomete principalmente homens, brancos com mais de 50 anos e vem crescendo sua incidência no ocidente. A prevalência na população geral não é precisamente conhecida, mas alguns estudos sugerem variar de 5 a $9 \%$ na população geral $1^{9,12,25}$. Estudo prévio evidenciou que a incidência é de 22,4 casos $/ 100.000$ habitantes ou $3,57 \%$ dos pacientes com DRGE ${ }^{1}$.

A metaplasia intestinal é considerada lesão pré-maligna com evolução imprevisível para adenocarcinoma ${ }^{6}$. A sequência de eventos é bem conhecida e caracterizada por epitélio normal - esofagite - metaplasia (EB) - displasia de baixo grau - displasia de alto grau - adenocarcinoma ${ }^{19}$.

Enquanto a metaplasia é a substituição do tecido epitelial escamoso pelo colunar intestinal, a displasia consiste

Trabalho realizado na Faculdade de Medicina de Jundiaí, Jundiaí, SP, Brasil

Correspondência: Sérgio Ferreira Módena, e-mail: smodena@dglenet.com.br em mudança da arquitetura celular. No EB com displasia de baixo grau, não há perda de polaridade dos núcleos, há redução do número de células caliciformes, porém o estroma entre as células está presente. A perda da maturação epitelial é caracterizada pela presença de núcleos proporcionalmente maiores na superfície da mucosa. A displasia de alto grau, a perda de maturação epitelial é evidente. Há alteração da arquitetura da mucosa com ramificação das criptas e superfície viliforme. Os núcleos têm maior pleomorfismo e hipercromasia, as alterações histológicas são similares ao carcinoma intramucoso ${ }^{24}$.

A diferenciação entre a displasia baixo grau e a de alto grau depende da distribuição do núcleo, que permanece confinado na metade basal das células nas denominadas displasias de baixo grau e que se apresenta distribuído aleatoriamente entre as metades basal e apicular nas de alto grau. A diferenciação entre displasia de alto grau e carcinoma "in situ" é que na displasia de alto grau, existe respeito à lamina própria celular, enquanto que no carcinoma "in situ", corrompe a membrana basal e invade a lâmina própria?.

Os sais de nitrito são encontrados na natureza e conhecidos há séculos pelo seu poder de conservar alimentos, principalmente carnes defumadas e vegetais. A matéria prima para sua origem são os nitratos, os quais sob a ação 
de algumas enzimas produzidas por bactérias comuns no trato digestivo, transformam-se em nitritos e adquirem sua forma ativa ${ }^{23,29}$.

$\mathrm{O}$ interesse pelo estudo dos compostos nitrosos cresceu depois do trabalho clássico de Sugimura et al. ${ }^{28}$ que conseguiu induzir adenocarcinoma na porção glandular do estômago de ratos após o uso de N-Methyl-N-Nitro-N-Nitrosoguanidine, mimetizando a doença humana. Além disso, pela alta incidência de carcinoma gástrico em regiões ricas em nitrito na água potável e no solo de algumas regiões, como Chile e Colômbia e também no Irã onde as carnes defumadas, sobremaneira peixe, são largamente consumidos ${ }^{13,14}$.

Alguns modelos experimentais utilizando animais de laboratório foram empregados para produzir adenocarcinoma na transição esôfago gástrica e esôfago de Barrett com ou sem uso de carcinógenos ${ }^{2,11,22}$. Chen et al. ${ }^{4}$ conseguiram induzir carcinoma em modelo experimental de refluxo gastroesofágico em ratos realizando anastomose duodenogástrica látero-lateral, associando o uso de ferro por via intramuscular. Os animais toleraram bem o procedimento, o qual tem reprodutibilidade em laboratório.

Não foi encontrada na literatura pesquisa experimental utilizando os nitritos associados a modelo experimental para indução de refluxo gastroesofágico e sua associação com EB e adenocarcinoma nesta região do trato digestivo, motivo pelo qual esta pesquisa foi realizada.

\section{MÉTODOS}

O experimento foi realizado em conformidade com os Princípios Éticos na Experimentação Animal adotados pelo Colégio Brasileiro de Experimentação Animal (COBEA) e aprovado pela Comissão de Ética na Experimentação Animal (CEEA) da UNICAMP.

Sessenta ratos Wistar (Rattus norvergicus albinus, Rodentia mammalia), considerados saudáveis e livres de doenças específicas (SPF - Specific Pathogen Free), com aproximadamente três meses de vida, machos e com peso aproximado de $250 \mathrm{~g}$, foram utilizados na pesquisa. Durante o experimento, os animais foram acondicionados separadamente em gaiolas próprias, mantidos em temperatura ambiente sob fluxo de ar contínuo com umidade relativa do ar controlada em 50\%, com ciclos diurnos de luz e alimentados com ração sólida para a raça e água tratada ad libitum, sendo observados diariamente, verificando-ae possíveis alterações comportamentais ou fisiológicas.

Vinte animais foram separados em dois grupos controles de 10 animais cada. $O$ primeiro (G1) recebeu dieta padrão e água filtrada. O segundo (G2) recebeu dieta padrão e água filtrada contendo solução de nitrito de sódio na proporção de $0,75 \mathrm{mg} / \mathrm{mL}$ durante 42 semanas.

Quarenta animais (Grupos G3 e G4) após 12 horas de jejum foram anestesiados com pentobarbital sódico (30 mg/Kg IV) através da veia dorsal da cauda. Após a tricotomia, assepsia da parede abdominal com solução iodada e laparotomia mediana, foi realizada anastomose duodenogastroesofágica látero-lateral, empregando fio de prolene 5-0 (Figura 1).

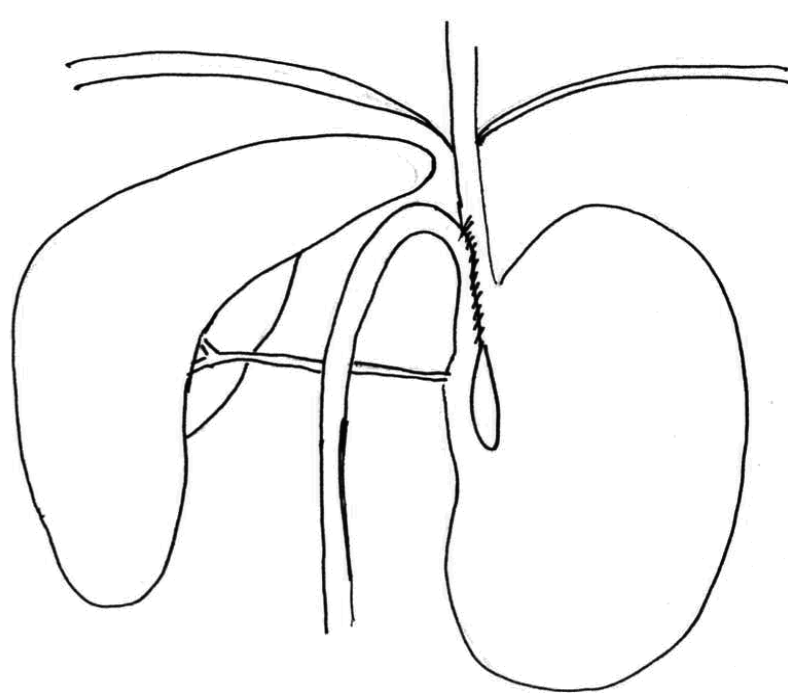

FIGURA 1 - Esquema da operação duodenogastroesofagoanastomose látero-lateral

Vinte animais operados (Grupo G3) voltaram a receber água filtrada 12 horas após a operação e ração padrão após 24 horas, sendo observados também por 42 semanas. Neste grupo um animal morreu de causa desconhecida.

Os demais 20 animais operados (Grupo G4) voltaram a receber água filtrada 12 horas após a operação e ração padrão após 24 horas. A partir do $3^{\circ}$ dia de pós-operatório tiveram oferta líquida de solução de água filtrada com nitrito de sódio na proporção de $0,75 \mathrm{mg} / \mathrm{mL}$, durante 42 semanas. Neste grupo, dois animais morreram de causa desconhecida.

Finalizado o tempo de observação, os animais foram relaparotomizados, submetidos à eutanásia, e removidas a transição esôfago-gástrica onde a anastomose havia sido previamente realizada, para realização de estudos histopatológicos.

As peças foram fixadas em formalina tamponada a $10 \%$, incluídos em blocos de parafina e submetida a cortes de 5 $\mathrm{mm}$ em micrótomo, coradas pela técnica de HematoxilinaEosina. Os estudos histopatológicos incluíram análise da intensidade da esofagite, a metaplasia intestinal, a displasia, esôfago de Barrett e o adenocarcinoma. Os critérios para laudo histopatológico utilizados foram da Classificação de Viena ${ }^{27}$ resumidos no Quadro 1.

\begin{tabular}{|l|l|}
\hline CATEGORIA 1 & $\begin{array}{l}\text { Negativo para displasia. Arquitetura dentro dos limites da } \\
\text { normalidade. Ausência de anormalidades nucleares, exceto } \\
\text { estratificação nuclear. Maior alteração nuclear quando as- } \\
\text { sociado com inflamações ou ulcerações }\end{array}$ \\
\hline CATEGORIA 2 & $\begin{array}{l}\text { Indefinido para displasia. A arquitetura pode estar distorcida } \\
\text { moderadamente. Alterações nucleares menos marcantes das } \\
\text { que são observadas na displasia }\end{array}$ \\
\hline CATEGORIA 3 & $\begin{array}{l}\text { Displasia de baixo grau (DBG). Arquitetura celular alterada } \\
\text { com núcleo se mantendo na metade basal das células }\end{array}$ \\
\hline CATEGORIA 4 & $\begin{array}{l}\text { Displasia de alto grau (DAG). Alterações severas, principal- } \\
\text { mente se envolvem a superfície mucosa, porém, respeitando } \\
\text { a lâmina própria }\end{array}$ \\
\hline CATEGORIA 5 & $\begin{array}{l}\text { Neoplasia invasiva. O carcinoma invade através da mem- } \\
\text { brana basal das glândulas na lâmina própria, não invadindo } \\
\text { a submucosa }\end{array}$ \\
\hline
\end{tabular}

QUADRO 1 - Classificação de Viena para laudos histopatológicos 
Os resultados obtidos foram analisados estatisticamente empregando os testes de comparações múltiplas de Tukey, e o exato de Fisher, expressos em porcentuais e mostrados em tabelas e gráficos $(P<0,05)$.

\section{RESULTADOS}

A Tabela 1 e o Gráfico 1 resumem a intensidade da esofagite conforme os grupos.

TABELA 1 - Intensidade da esofagite conforme os grupos

\begin{tabular}{|c|c|c|c|c|c|c|c|c|c|}
\hline \multirow[b]{2}{*}{ ESOFAGITE } & \multicolumn{2}{|c|}{$\begin{array}{c}\text { G1 } \\
\text { (10 animais) }\end{array}$} & \multicolumn{2}{|c|}{$\begin{array}{c}\text { G2 } \\
\text { (10 animais) }\end{array}$} & \multicolumn{2}{|c|}{$\begin{array}{c}\text { G3 } \\
\text { (19animais) }\end{array}$} & \multicolumn{2}{|c|}{$\begin{array}{c}\text { G4 } \\
\text { (18 animais) }\end{array}$} & \multirow{2}{*}{$\begin{array}{l}\boldsymbol{P} \\
<0.0001\end{array}$} \\
\hline & $\mathrm{n}$ & $\%$ & $\mathrm{n}$ & $\%$ & $\mathrm{n}$ & $\%$ & $\mathrm{n}$ & $\%$ & \\
\hline AUSENTE & 10 & 100.0 & 10 & 100.0 & 6 & 31.6 & 8 & 44.4 & \\
\hline LEVE & 0 & 0.0 & 0 & 0.0 & 9 & 47.4 & 1 & 5.6 & \\
\hline MODERADA & 0 & 0.0 & 0 & 0.0 & 2 & 10.5 & 0 & 0.0 & \\
\hline INTENSA & 0 & 0.0 & 0 & 0.0 & 2 & 10.5 & 9 & 50.0 & \\
\hline COMPARAÇÕES & \multicolumn{2}{|r|}{$\mathrm{P}$} & & & & & & & \\
\hline $\mathrm{G} 3 \times \mathrm{G} 2$ & \multicolumn{2}{|c|}{0.0012} & \multicolumn{2}{|c|}{$P<0,05$} & & & & & \\
\hline $\mathrm{G} 4 \times \mathrm{G} 2$ & \multicolumn{2}{|c|}{0.0035} & \multicolumn{2}{|c|}{$P<0,05$} & & & & & \\
\hline G3 x G4 & 0.0 & & \multicolumn{2}{|c|}{$P<0,05$} & & & & & \\
\hline
\end{tabular}

Nota-se que no grupo de animais não operados não foi registrado nenhum com esofagite. Porém no grupo de operados, com e sem ingestão de nitrito de sódio, foi registrado esofagite em intensidades variáveis. No G3 a esofagite foi ausente em $31,6 \%$ dos animais, sendo leve e moderada na maioria deles $(57,9 \%)$ e intensa e apenas $10,5 \%$. Por outro lado, no G4 a esofagite encontrada foi intensa em $50 \%$ dos animais.

Foram observadas diferenças estatísticas significativas entre os grupos estudados (Tabela 1).

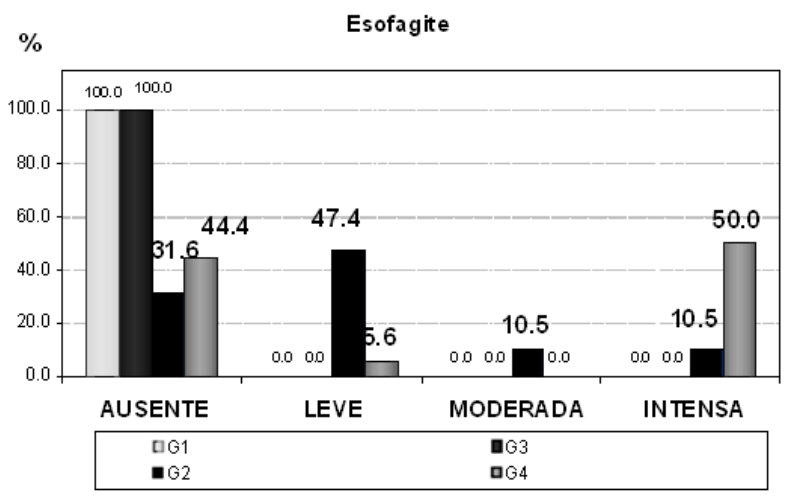

GRÁFICO 1 - Distribuição percentual de esofagite segundo grupo de estudo

A Tabela 2 e o Gráfico 2 resumem a metaplasia intestinal encontrada nos grupos.
TABELA 2 - A metaplasia intestinal segundo os grupos

\begin{tabular}{|c|c|c|c|c|c|c|c|c|c|}
\hline & \multicolumn{2}{|c|}{$\begin{array}{c}\text { G1 } \\
\text { (10 animais) }\end{array}$} & \multicolumn{2}{|c|}{$\begin{array}{c}\text { G2 } \\
\text { (10 animais) }\end{array}$} & \multicolumn{2}{|c|}{$\begin{array}{c}\text { G3 } \\
\text { (19animais) }\end{array}$} & \multicolumn{2}{|c|}{$\begin{array}{c}\mathrm{G4} \\
\text { (18 animais) }\end{array}$} & $P$ \\
\hline METAPLASIA & & & & & & & & & $<0.0001$ \\
\hline Ausente & 10 & 100.0 & 10 & 100.0 & 14 & 73.7 & 13 & 72.2 & \\
\hline Presente & 0 & 0.0 & 0 & 0.0 & 5 & 26.3 & 5 & 27.8 & \\
\hline COMPARAÇÕES & & $\mathrm{P}$ & & & & & & & \\
\hline $\mathrm{G} 3 \times \mathrm{G} 2$ & 0.1 & & $(P>$ & $>0,05)$ & & & & & \\
\hline G4 x G2 & 0.0 & & $(P<$ & $0,05)$ & & & & & \\
\hline G3 x G4 & 0.0 & & $(P<$ & $00,05)$ & & & & & \\
\hline
\end{tabular}

Nota-se que nos animais não operados (G1 e G2) não foi encontrada metaplasia intestinal. Ela apenas foi registrada nos animais operados (G3 e G4), respectivamente, 26,3\% e $72,2 \%$. Foram observadas diferenças estatisticamente significativas entre os grupos, sendo que na comparação G3 versus $\mathrm{G} 4$ o valor de $\mathrm{P}$ foi muito significativo $(P=0.0001$ $-P<0,05)$ (Tabela 2).

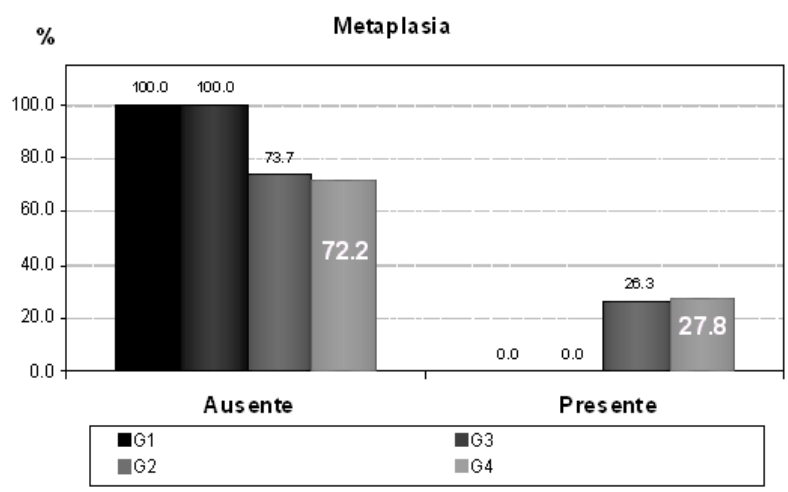

GRÁFICO 2 - Distribuição percentual de metaplasia intestinal conforme os grupos de estudo

Foram observadas diferenças estatisticamente significativas entre os grupos em relação à ocorrência do Esôfago de Barrett $(P<0,05)$, o qual foi encontrado sem displasia e com displasia apenas nos grupos operados. No grupo G3 foi registrado em 26,3\% (5/19) dos animais, enquanto no grupo G4 em 72,3\% (13/18). No grupo G4 foram encontrados seis animais com adenocarcinomas (33,33\%), associados à displasia de alto grau. Neste grupo também foram encontrados cinco animais com displasia indefinida $(27,8 \%)$. Entre os grupos G3 e G2 não houve diferença significativa nos estudos histopatológicos $(P>0,05)$ (Tabela 3 e Figura 3).

Quanto a Classificação de Viena, foram constatadas diferenças significativas entre os grupos. As categorias 2, 3 e 5 somente foram encontradas nos animais operados que ingeriram nitrito de sódio. Os grupos G1, G2 e G3 concentraram-se na categoria 1 de Viena (Tabela 4 e Gráfico 4). 
TABELA 3 - O esôfago de Barrett, a displasia e o adenocarcinoma nos grupos e análise estatística comparativa

\begin{tabular}{llllllllll}
\hline & $\begin{array}{l}\text { G1 } \\
\text { (10 animais) }\end{array}$ & $\begin{array}{l}\text { G2 } \\
\text { (10 animais) }\end{array}$ & $\begin{array}{l}\text { G3 } \\
\text { (19 animais) }\end{array}$ & $\begin{array}{l}\text { G4 } \\
\text { (18 animais) }\end{array}$ & $P$ \\
\hline $\begin{array}{l}\text { Estudos histopatológicos } \\
\text { Sem Barrett }\end{array}$ & 10 & $(100.0 \%)$ & 10 & $(100.0 \%)$ & 14 & $(73.7 \%)$ & 5 & $(27.8 \%)$ & \\
$\begin{array}{l}\text { Barrett sem } \\
\text { displasia }\end{array}$ & 0 & 0.0 & 0 & 0.0 & 5 & $(26.3 \%)$ & 1 & $(5.6 \%)$ & \\
Barrett + DBG & 0 & 0.0 & 0 & 0.0 & 0 & 0.0 & 1 & $(5.6 \%)$ \\
$\begin{array}{l}\text { Barrett + displa- } \\
\text { sia indefinida }\end{array}$ & 0 & 0.0 & 0 & 0.0 & 0 & 0.0 & 5 & $(27,8 \%)$ \\
$\begin{array}{l}\text { Barrett + DAG+ }+ \\
\text { adenocarcinoma* }\end{array}$ & 0 & 0.0 & 0 & 0.0 & 0 & 0.0 & 6 & $(33,3 \%)$ \\
$\begin{array}{l}\text { Estudos estatísticos } \\
\text { G3 x G2 }\end{array}$ & $P$ & & & & & & & & \\
G4 x G2 & $0.1336(\mathrm{P}>0,05)$ & $P<0,05$ & & & & & \\
G3 x G4 & $0.0031(\mathrm{P}<0,05)$ & $P<0,05$ & & & & & \\
\hline
\end{tabular}

OBS: os resultados histopatológicos do G2 foram idênticos ao G1

* um adenocarcinoma moderadamente diferenciado invadido até muscular, quatro moderadamente diferenciados invadido adventícia e um mucinoso invadido adventícia

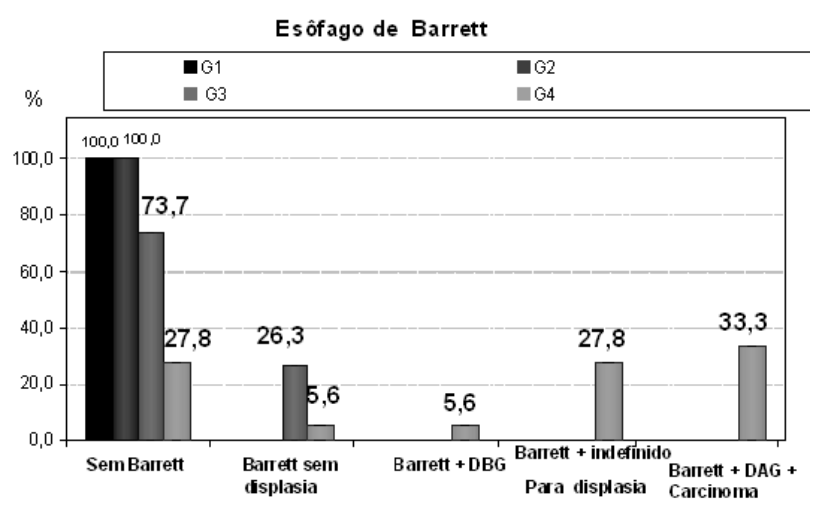

GRÁFICO 3 - Comparações percentuais dos estudos histopatológicos entre os grupos.

\section{DISCUSSÃO}

O adenocarcinoma da transição esôfago-gástrica é a neoplasia que apresentou maior elevação de incidência na última década e o seu prognóstico em cinco anos é pobre se o diagnóstico e tratamento não forem instituídos precocemente ${ }^{10}$. Portanto, é muito importante entender o processo da doença e instituir mecanismos para a sua prevenção.

$\mathrm{O}$ refluxo gastroesofágico, principalmente a combinação de conteúdo gástrico e duodenal é bem conhecido como fator de risco para adenocarcinoma da transição esôfago-gástrica. Os indivíduos que apresentam refluxo gastroesofágico crônico evoluem para o aparecimento do esôfago de Barrett. O estilo de vida, os hábitos do tabagismo e etilismo são fatores importantes envolvidos ${ }^{8}$.

Estudos recentes têm demonstrado que o adenocarcinoma tem como precursor a displasia e, paralelamente à sequência metaplasia-displasia- adenocarcinoma, pesquisas têm demonstrado mudanças genéticas e moleculares nas células, motivados por diversos fatores, tais como ativação
TABELA 4 - Classificação de Viena, conforme os grupos de estudo e análise estatística comparativa

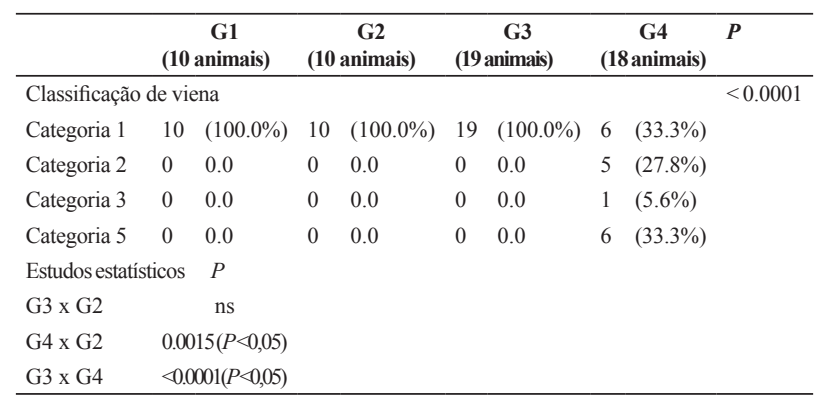

ns = não significante

Classificação de Viena

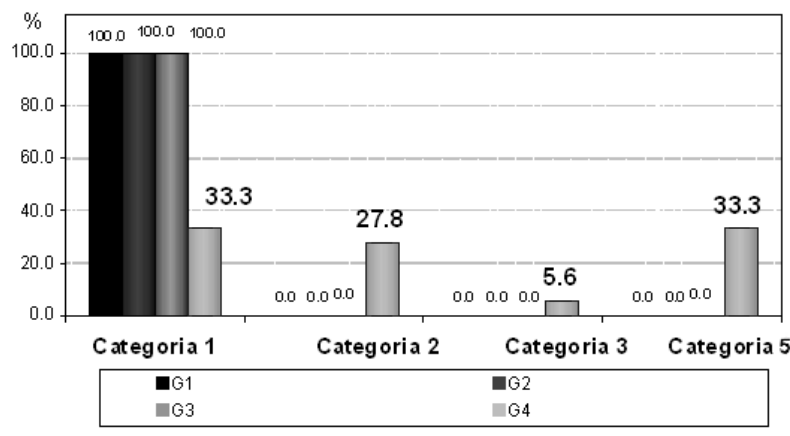

GRÁFICO 4 - Distribuição percentual de categorias da Classificação de Viena, segundo os grupos de estudo

de oncogenes específicos, inibição de genes supressores tumores, além de fatores ambientais ${ }^{3,6,16}$.

A displasia é definida como inequivoca condição prémaligna e a severidade das anormalidades citológicas e arquiteturais são utilizadas como classificação para a sua graduação. As classificações de displasia no esôfago de Barrett são adaptações das utilizadas em outras porções do trato gastrointestinal: leve, moderada ou grave ${ }^{9}$. Frequentemente há discrepâncias entre os patologistas ocidentais e orientais para classificar o grau de displasia no esôfago de Barrett e por este motivo é que foi proposta em 1998 a Classificação de Viena em cinco categorias ${ }^{6,9,20,27}$. Posteriormente esta classificação foi revisada em $2002^{5}$.

As vantagens na sua utilização são que emprega a terminologia displasia habitualmente mais utilizada por patologistas ocidentais, substituindo a neoplasia, e ordena os diagnósticos em grupos de categorias numeradas de 1 a 5. Além disso, o diagnóstico de carcinoma invasivo (categoria 5) requer a constatação que ocorreu invasão de no mínimo a lâmina própria da mucosa. Desse modo, a Classificação de Viena é prática, permite distinguir com mais detalhes a graduação da displasia, entretanto, ainda não está em uso corrente na prática diária e é preciso ainda alguns anos para comprovação se é efetiva na condução e tratamento dos doentes ${ }^{26}$. 
Na presente pesquisa, o emprego desta classificação foi muito útil para caracterizar a evolução das alterações citoarquiteturais, nos animais não operados, desde a metaplasia, passando por displasia de baixo grau, de alto grau, até o adenocarcinoma invasivo. A displasia de algo grau (categoria 4) não foi identificada isoladamente, apenas identificada associada ao adenocarcinoma. A incidência de alterações indefinidas para displasia (categoria 2) foi de $27,8 \%$. Entretanto, permitiu com facilidade a identificação da displasia de baixo grau (categoria 3 ) e do adenocarcinoma invasivo (categoria 5).

Os modelos experimentais de refluxo gástrico e duodenal para produzir esôfago de Barrett e o adenocarcinoma no esôfago são muito conhecidos há anos, utilizando ou não carcinógenos. Alguns autores preferiram utilizar a gastrectomia total ${ }^{11,21,22} \mathrm{e}$ outros realizar procedimentos que facilitem o refluxo gástrico, biliar ou bílio-pancreático ao esôfago ${ }^{2,4,18,21}$. Attwood et al. ${ }^{2}$, utilizaram em um grupo de ratos Sprague-Dawley a anastomose duodenogastroesofágica, semelhante a que foi utilizada nesta pesquisa e, observando os animais durante 30 semanas, registraram apenas um animal com adenocarcinoma. Entretanto, quando associaram carcinógenos (2-6-dimethylnitrosomorproline e methyl-n-amylnitrosamine) a outro grupo de animais, também operados pela mesma técnica, evidenciou-se elevada ocorrência tanto de carcinoma espinocelular como de adenocarcinoma.

Goldstein et al. ${ }^{11}$ e Chen et al. ${ }^{4}$ utilizaram modelos experimentais de refluxo misto (gástrico e duodenal), sem o emprego de carcinógenos, administrando ferro aos animais, respectivamente, durante 30 e 40 semanas e obtiveram esôfago de Barrett com sem displasia e adenocarcinomas associados. Os dois autores registraram adenocarcinomas mucinosos bem diferenciados e concluíram que os modelos experimentais são consistentes com o que é proposto para a sequência humana de eventos patológicos de progressão do esôfago de Barrett, para esôfago de Barrett com displasia e então o adenocarcinoma.

A comparação entre este trabalho e os demais que empregaram modelos experimentais semelhantes, é difícil devido às variáveis envolvidas, tais como: o número de animais, raça, tempo de observação, tipo do refluxo, carcinógeno utilizado e a via de administração. Por outro lado, quando se considera o uso ou não de carcinógeno, fica evidente que a administração do carcinógeno aumenta a incidência de adenocarcinoma, independente da via de administração.

$\mathrm{Na}$ presente pesquisa, o modelo experimental empregado com ampla anastomose duodenogastroesofágica, e observação dos animais durante 42 semanas, sem dúvida propiciou refluxo de conteúdo gástrico e duodenal (biliar e pancreático) ao esôfago causando importantes alterações citoarquiteturais na mucosa. A associação entre a operação, que facilita o refluxo misto ao esôfago, e o nitrito de sódio promoveu intensa reação tecidual com esofagite ulcerativa, metaplasia intestinal, displasia de baixo e de alto grau e adenocarcinoma invasivo. A metaplasia intestinal foi encontrada apenas no grupo de animais operados, sendo que no de animais que ingeriu nitrito foi mais prevalente (G4). $\mathrm{O}$ adenocarcinoma foi registrado em $33,3 \%$ dos animais operados e que ingeriram nitrito de sódio, correspondendo à categoria 5 da Classificação de Viena, sendo que os tipos histológicos foram cinco adenocarcinomas moderadamente diferenciados e um adenocarcinoma mucinoso. Mais uma vez comprovou-se que este tipo de refluxo é responsável pela etiopatogenia do esôfago de Barrett. Salienta-se que o grupo de animais não operados e que também ingeriram nitritos não desenvolveram o esôfago de Barrett.

Pesquisas avançadas dos mecanismos moleculares e celulares de carcinogênese têm sido concentradas nos compostos n-nitrosos, na tentativa de descobrir porque e como estas drogas produzem tumores em grande número de espécies animais com especificidade celular e orgânica. Os compostos n-nitrosos são hoje considerados um grupo de substâncias orgânicas resultantes da interação entre os nitritos e aminoácidos presentes nos alimentos ingeridos, formando as nitrosaminas. Esta nitrosação pode ocorrer nos alimentos, no interior do trato digestivo e in vitro ${ }^{15,17,29}$.

Os nitritos e nitratos de sódio e potássio são substâncias inorgânicas encontradas na natureza, em uma grande variedade de alimentos consumidos pelo homem, na água potável e nos fertilizantes. São amplamente utilizados na indústria alimentícia como conservantes de carnes, enlatados e produtos defumados ${ }^{14,15,17,29}$. Portanto, o consumo exagerado destes produtos, certamente eleva a concentração de nitritos no trato digestivo alto, os quais associados ao refluxo gastroesofágico misto, podem perfeitamente contribuírem para a etiopatogenia do esôfago de Barrett, e a subsequente evolução para displasia e adenocarcinoma. Novas pesquisas são necessárias para verificar a relação entre a ingestão de nitritos, formação de compostos nnitrosos e a etiopatogenia do esôfago de Barrett, displasia e adenocarcinoma.

Os resultados desta pesquisa indicam que as mudanças morfológicas características de progressão do esôfago de Barrett ao adenocarcinoma neste modelo animal são similares às registradas na literatura para humanos, ou seja, $o$ refluxo dos conteúdos gástrico e duodenal causando a metaplasia intestinal, em seguida progredindo para displasia de baixo grau, displasia de alto grau e o adenocarcinoma.

\section{CONCLUSÃo}

O refluxo misto, gástrico e duodenal é o principal fator para a origem do adenocarcinoma na transição esôfagogástrica, e a possível prevenção estaria nos hábitos alimentares, evitando-se alimentos contendo conservantes e optando por alimentação cozida recente, frutas e legumes. 
Modena SF, Meirelles LR, Araujo MR. Are the nitrites important in a genesis of the adenocarcinoma associated to the Barrett esophagus? ABCD Arq Brás Cir Dig 2008;21(3):124-9

ABSTRACT - Background - Barrett's esophagus is a complication of gastroesophageal reflux and predisposes to adenocarcinoma of the esophagogastric transition and several factors have been related to its pathogenesis. Aim - To evaluated the genesis of adenocarcinoma associated with Barrett's esophagus in an experimental model of duodenogastroesophageal reflux with the additional ingestion of sodium nitrite Methods - Sixty male Wistar rats were divided into four groups. Twenty of these animals served as controls (10 animals ingesting only water and 10 ingesting water plus a solution of sodium nitrite), while the remaining 40 animals were submitted to side-to-side duodenogastroesophageal anastomosis (20 animals ingesting only water and 20 ingesting water plus the sodium nitrite solution). The occurrence of esophagitis, metaplasia, dysplasia, Barrett's esophagus associated with adenocarcinoma were analyzed. The Vienna classification for dysplasia and adenocarcinoma was used in the analysis of results. Results - After 42 weeks of observation, Barrett's esophagus was found in $26.3 \%(5 / 19)$ of the animals only submitted to surgery compared to $72.3 \%(13 / 18)$ of the animals in the group submitted to surgery and given nitrites. Six cases of adenocarcinoma (33.3\%) were also found in this latter group. Categories 2, 3 and 5 of the Vienna classification were only found in animals submitted to surgery plus sodium nitrite (66.7\%). Conclusion - The ingestion of sodium nitrite associated with duodenogastroesophageal reflux plays an important role in the genesis of adenocarcinoma associated with Barrett's esophagus.

HEADINGS - Duodenogastric reflux. Nitrites. Barrett Esophagus. Adenocarcinoma.

\section{REFERÊNCIAS}

1. Andreollo NA, Michelino MU, Brandalise NA, Lopes LR, Trevisan MA, Leonardi LS. Incidence and epidemiology of Barrett's epitellium at the Gastrocentro-Unicamp. Arq Gastroenterol, 1997;34:22-6.

2. Attwood SE, Smyrk TC, DeMeester TR, Mirvish SS, Stein HJ, Hinder RA. Duodenoesophageal reflux and the development of esophageal adenocarcinoma in rats. Surgery. 1992;111(5):503-10.

3. Chandrasoma P, Wickramasinghe K, Ma Y, DeMeester T. Is intestinal metaplasia a necessary precursor lesion for adenocarcinomas of the distal esophagus, gastroesophageal junction and gastric cardia? Dis Esophagus. 2007;20, 36-41.

4. Chen X, Guang-yu Y, Wei YD, Flordeliza B, Stephen KC, Chung SY. An esophagogastroduodenal anastomosis model for esophageal adenocarcinogenisis in rats enhancement iron overload. Cacinogenesis, $1999: 20(9): 1801-08$

5. Dixon MF. Gastrointestinal epithelial neoplasia: Vienna revisited. Gut. 2002;51:130-1.

6. Fléjou J. Barret's esophagus: from metaplasia to dysplasia and cancer. Gut 2005;54(Suppl I):i6-i12.

7. Fléjou, J-F \& Svrcek M. Barret's oesophagus - a pathologist's view. Histopatology. 2007; 50, 3-14.

8. Gatenby PA, Caygill CP, Ramus JR, Charlett A, Watson A. Barrett's columnarlined oesophagus: demographic and lifestyle associations and adenocarcinoma risk. Dig Dis Sci. 2008 ;53(5):1175-85.

9. Geboes K \& Van Eyken P. The diagnosis of dysplasia and malignancy in Barrett's oesophagus. Histopathology 2000;37:99-107.

10. Gerson LB, Triadafilopoulos G. Screening for esophageal adenocarcinoma: an evidence-based approach. Am J Med. 2002;113(6):499-505.

11. Goldstein SR Development of esophageal metaplasia and adenocarcinoma in a rat surgical model without the use of carcinogen. Carcinogenesis, 1997 Nov;18(11):2265-70.

12. Hirota WK, Loughney TM, Lazas DJ, Maydonovithe CL, Rholl V, Wong RKH. Specialized intestinal metaplasia, dysplasia and cancer of the esophagus and esophagogastric junction: prevalence and clinical data. Gastroenterology 1999; 116:227-285

13. Hotchkiss JH, A Review of Current Literature on N-Nitroso Compounds in Foods. Adv. Food Res., 31: 55-115, 1987.

14. Jakszyn P, Agudo A, Berenguer A, Ibáñez R, Amiano P, Pera G, Ardanaz E, Barricarte A, Chirlaque MD, Dorronsoro M, Larrañaga N, Martinez C, Navarro C, Quirós JR, Sanchéz MJ, Tormo MJ, González CA. Intake and food sources of nitrites and N-nitrosodimethylamine in Spain. Public Health Nutr. 2006;9(6):785-91.

15. Jakszyn P, Gonzalez CA. Nitrosamine and related food intake and gastric and oesophageal cancer risk: a systematic review of the epidemiological evidence. World J Gastroenterol. 2006;12(27):4296-303.
16. Kerkhof M, Kusters JG, van Dekken H, Kuipers EJ, Siersema PD. Biomarkers for risk stratification of neoplastic progression in Barrett esophagus. Cell Oncol. 2007;29(6):507-17.

17. Lijinsky W. N-Nitroso compounds in the diet. Mutat Res. 1999;443(1-2):12938.

18. Miwa K Reflux of duodenal or gastro-duodenal contents induces esophageal carcinoma in rats. Int. J. Cancer 1996:67,269-274

19. Oberg S, Wenner J, Johanson J, Walther B. Barrett's esophagus risk factors for progression to dysplasia and adenocarcinoma. Ann Surg. 2005;242(1):49-54.

20. Odze RD. Diagnosis and grading of dysplasia in Barrett's oesophagus. J Clin Pathol. 2006;59:1029-1038.

21. Pera M, Grande L, Gelabert M. et al Epithelial cell hyperproliferation after biliopancreatic reflux into esophagus of rats. Ann Thorac Surg 1998;65:779-86

22. Pera M, Trastek VF, Carpenter HA, Fernandez PL, Cardesa A, Mohr U, Pairolero PC. Influence of pancreatic and biliary reflux in the development of esophageal carcinoma. Ann Thorac Surg. 1993:55(6):1386-93.

23. Reed PI. The role of nitrosamines in cancer formation. Bibl Nutr Dieta. 1986;(37):130-8.

24. Rodrigues M.A.M. Barret's esophagus and dysplasia: diagnostic criteria. J Bras Patol Med Lab. 2004; 40(3): 185-191.

25. Rokainen J., Aro P., Storkrubb T. et al. Prevalence of Barrett's Esophagus in the General Population: an Endoscopic Study. Gastroenterology 2005: 129; 1825-31.

26. Salas Caudevilla A. Evaluation of dysplasia in gastrointestinal diseases. Gastroenterol Hepatol. 2007;30(10):602-11

27. Schlemper RJ, Riddell RH, Kato Y, Borchard F, Cooper HS, Dawsey SM, Dixon MF, Fenoglio-Preiser CM, Fléjou JF, Geboes K, Hattori T, Hirota T, Itabashi M, Iwafuchi M, Iwashita A, Kim YI, Kirchner T, Klimpfinger M, Koike M, Lauwers GY, Lewin KJ, Oberhuber G, Offner F, Price AB, Rubio CA, Shimizu M, Shimoda T, Sipponen P, Solcia E, Stolte M, Watanabe H, Yamabe $\mathrm{H}$. The Vienna classification of gastrointestinal epithelial neoplasia. Gut. 2000;47(2):251-5

28. Sugimura T, Fujimura S, Baba T. Tumor production in the glandular stomach and alimentary tract of the rat by N-Methyl-N-Nitro-N-Nitrosoguanidine. Cancer Res. 1970;30:455-65.

29. Ward MH, Heineman EF, Markin RS, Weisenburger DD. Adenocarcinoma of the stomach and esophagus and drinking water and dietary sources of nitrate and nitrite. Int J Occup Environ Health. 2008;14(3):193-7.

Fonte de financiamento: não há Conflito de interesse: não há Recebido para publicação: 01/03/2008 Aceito para publicação: 30/05/2008 